\title{
Symmetries of the space of connections on a principal $G$-bundle and related symplectic structures
}

\author{
Grzegorz Jakimowicz, Anatol Odzijewicz, Aneta Sliżewska
}

October 9, 2018

\section{Contents}

1 Introduction 1

2 Symmetries of the tangent bundle of a principal bundle 2

3 The action of $A u t_{T G} T P$ on the space of connections

4 Space of generalized canonical forms on $T^{*} P$

5 The Marsden-Weinstein reduction $\quad 15$

\begin{abstract}
We investigate $G$-invariant symplectic structures on the cotangent bundle $T^{*} P$ of a principal $G$-bundle $P(M, G)$ which are canonically related to automorphisms of the tangent bundle $T P$ covering the identity map of $P$ and commuting with the action of $T G$ on $T P$. The symplectic structures corresponding to connections on $P(M, G)$ are also investigated. The Marsden-Weinstein reduction procedure for these symplectic structures is discussed.
\end{abstract}

\section{Introduction}

The phase space of a typical Hamiltonian system is the cotangent bundle $T^{*} P$ of its configurations space $P$ equiped with the standard symplectic form. Usually one considers the case when Hamiltonian of this system is invariant with respect to the cotangent lift of an action of some group $G$ on $P$. Therefore, it is sometimes reasonable to replace the standard symplectic form with another $G$-invariant symplectic form on $T^{*} P$ which retains certain properties. Assuming that the action of $G$ on $P$ is free and the quotient space $P / G$ is a manifold $M$ one can consider $P$ as the total space of the principal $G$-bundle $P(M, G)$. Motivated by the above we consider here the following structures related to $P(M, G)$ in a natural way. 
(i) The space $\operatorname{CanT}^{*} P$ of fibre-wise linear non-singular differential one-forms $\gamma$ on the cotangent bundle $T^{*} P$, which annihilate the vectors tangent to the fibres of $T^{*} P$.

(ii) The space $\operatorname{Conn} P(M, G)$ of connections on the principal $G$-bundle $P(M, G)$.

(iii) The group $A u t_{T G} T P$ of automorphisms of the tangent bundle $T P$ covering the identity map of $P$ which commute with the action of the tangent group TG on TP.

Since the canonical one-form $\gamma_{0}$ belongs to $\operatorname{Can} T^{*} P$ and other elements of $\operatorname{CanT} T^{*} P$ posses some properties of $\gamma_{0}$, hence they are called generalized canonical forms here.

There are important relations between the above structures. Namely the group $A u t_{T G} T P$ acts on the both subspaces mentioned above. The action on $\operatorname{ConnP}(M, G)$ is transitive and the action on $\operatorname{CanT}^{*} P$ is free. The orbit $\operatorname{Can}_{T G} T^{*} P$ of $A u t_{T G} T P$ through the canonical form $\gamma_{0}$ consists of $G$-invariant generalized canonical forms $\gamma$ such that the momentum maps corresponding to symplectic forms $\omega=d \gamma$ coincide with the momentum map which corresponds to the standard symplectic form $\omega_{0}=d \gamma_{0}$. In this way one obtains a family of symplectic forms $\omega_{A}$ on $T^{*} P$ enumerated by the elements $A$ of the group $A u t_{T G} T P$.

The group $A u t_{T G} T P$ is investigated in Section 2, see Proposition 2.

In Section 3 we describe the action of the group $A u t_{T G} T P$ on $C o n n P(M, G)$ and show that $A u t_{T G} T P$ can be defined equivalently as the group of symmetries of $\operatorname{Conn} P(M, G)$, see Proposition 4 .

The relations between the group $A u t_{T G} T P$ and the space $\operatorname{Can}_{T G} T^{*} P$ are investigated in Section 4, see Proposition 5 and Proposition 6. Among other things it is shown that fixing a reference connection $\alpha$ one embeds the space of all connections into the space $\operatorname{Can}_{T G} T^{*} P$ of generalized canonical forms, see Corrolary 7 . The choice of the reference connection $\alpha$ also allows ones to define a $G$-equivariant diffeomorphism $I_{\alpha}: T^{*} P \rightarrow$ $\bar{P} \times T_{e}^{*} G$, see [7, 12, where $\bar{P}$ is the total space of the pull back $\bar{P}\left(T^{*} M, G\right)$ of $P(M, G)$ on $T^{*} M$ and $T_{e}^{*} G$ is the dual of the Lie algebra $T_{e} G$. The generalized canonical forms and related symplectic forms $\omega_{A}$ written on $\bar{P} \times T_{e}^{*} G$ obtain the form consistent with the structure of the bundle $\bar{P} \times T_{e}^{*} G \rightarrow T^{*} M$, see (47) and (48).

In Section $\left[5\right.$ we discuss the $G$-Hamiltonian system on $\left(T^{*} P, \omega_{A}, J_{0}\right)$ which could be considered as a natural generalization of the ones investigated in [7, 11, 12]. The MarsdenWeinstein reduction procedure is applied to these systems.

\section{Symmetries of the tangent bundle of a principal bundle}

Let $P(M, G)$ be a principal bundle over a manifold $M$. Throughout the paper we will denote the right action of the Lie group $G$ on $P$ by $\kappa: P \times G \rightarrow P$ and write $\mu: P \rightarrow M$ for the bundle projection. We will use also the shorter notation $p g:=\kappa(p, g)$. For a fixed $p \in P$ and $g \in G$ one has the corresponding maps $\kappa_{p}: G \rightarrow P$ and $\kappa_{g}: P \rightarrow P$ defined by

$$
\kappa_{p}:=\kappa(p, \cdot) \quad \text { and } \quad \kappa_{g}:=\kappa(\cdot, g) .
$$


Recall that the tangent bundle $T G$ of $G$ is a Lie group itself with the product and the inverse defined as follows

$$
X_{g} \bullet Y_{h}:=T L_{g}(h) Y_{h}+T R_{h}(g) X_{g}, \quad X_{g}^{-1}:=-T L_{g^{-1}}(e) \circ T R_{g^{-1}}(g) X_{g},
$$

where $X_{g} \in T_{g} G, Y_{h} \in T_{h} G$ and $L_{g}(h):=g h, R_{g}(h):=h g$. Let $e \in G$ be the unit element of $G$ and $\mathbf{0}: G \rightarrow T G$ be the zero section of the tangent bundle $T G$. Then one has

$$
\begin{gathered}
X_{e} \bullet Y_{e}=X_{e}+Y_{e}, \quad \mathbf{0}_{g} \bullet \mathbf{0}_{h}=\mathbf{0}_{g h}, \\
X_{g} \bullet Y_{e} \bullet X_{g}^{-1}=\left(T R_{g^{-1}}(g) \circ T L_{g}(e)\right) Y_{e}=: A d_{g} Y_{e}
\end{gathered}
$$

So, the corresponding Lie algebra $T_{e} G$ can be considered as an abelian normal subgroup of $T G$ and the zero section $\mathbf{0}: G \rightarrow T G$ is a group monomorphism.

The diffeomorphism

$$
I: G \times T_{e} G \ni\left(g, X_{e}\right) \mapsto T R_{g}(e) X_{e}=: X_{g} \in T G
$$

allows us to consider $T G$ as the semidirect product $G \ltimes_{A d_{G}} T_{e} G$ of $G$ by the $T_{e} G$, where the group product of $\left(g, X_{e}\right),\left(h, Y_{e}\right) \in G \ltimes_{A d_{G}} T_{e} G$ is given by

$$
\begin{gathered}
\left(g, X_{e}\right) \bullet\left(h, Y_{e}\right)=I^{-1}\left(I\left(g, X_{e}\right) \cdot I\left(h, Y_{e}\right)\right)= \\
=\left(g h, X_{e}+T\left(R_{g^{-1}} \circ L_{g}\right)(e) Y_{e}\right)=\left(g h, X_{e}+A d_{g} Y_{e}\right) .
\end{gathered}
$$

Using the Lie group isomorphism (1) and the equality

$$
\kappa_{g} \circ \kappa_{p}=\kappa_{p} \circ R_{g}
$$

we obtain the action

$$
\Phi_{\left(g, X_{e}\right)}\left(v_{p}\right)=T \kappa_{g}(p)\left(v_{p}+T \kappa_{p}(e) X_{e}\right)
$$

of $G \ltimes_{A d_{G}} T_{e} G$ on the tangent bundle $T P$.

Applying the above action one obtains the following isomorphisms

$$
\begin{gathered}
T P / T^{v} P \cong T P / T_{e} G, \\
T P / T G \cong\left(T P / T_{e} G\right) / G \cong(T P / G) / T_{e} G, \\
T M=T(P / G) \cong T P / T G,
\end{gathered}
$$

of vector bundles, where we write $T^{v} P:=\operatorname{Ker} T \mu$ for the vertical subbundle of $T P$. These isomorphisms will be useful in subsequent considerations.

Another group important here which acts on $T P$ is the group $A u t_{0} T P$ of smooth automorphisms $A: T P \rightarrow T P$ of the tangent bundle covering the identity map of $P$, i.e. for any $p \in P$ one has the map $A(p): T_{p} P \rightarrow T_{p} P$ which is an isomorphism of the tangent space $T_{p} P$ and $A(p)$ depends smoothly on $p$. Note here that $A u t_{0} T P$ is a normal subgroup of the group AutTP of all automorphisms of $T P$. 
By $A u t_{T G} T P \subset A u t_{0} T P$ we denote the subgroup consisting of those elements of $A u t_{0} T P$ whose action on $T P$ commutes with the action (2) of $T G \cong G \ltimes_{A d_{g}} T_{e} G$ on $T P$, i.e.

$$
A(p g) \circ \Phi_{\left(g, X_{e}\right)}=\Phi_{\left(g, X_{e}\right)} \circ A(p) .
$$

From the isomorphisms (44) and (5) it follows that the group $A u t_{T G} T P$ acts also on vector bundles $T P / G \rightarrow M$ and $T M \rightarrow M$.

Proposition 1. $A \in A u t_{T G} T P$ if and only if

$$
\begin{gathered}
A(p) \circ T \kappa_{p}(e)=T \kappa_{p}(e) \\
A(p g) \circ T \kappa_{g}(p)=T \kappa_{g}(p) \circ A(p)
\end{gathered}
$$

for any $g \in G$ and $p \in P$.

Proof. Substituting (21) into (6) we obtain the equality

$$
A(p g)\left[T \kappa_{g}(p) v_{p}+\left(T \kappa_{g}(p) \circ T \kappa_{p}(e)\right) X_{e}\right]=T \kappa_{g}(p) A(p) v_{p}+\left(T \kappa_{g}(p) \circ T \kappa_{p}(e)\right) X_{e},
$$

which is valid for all $v_{p} \in T_{p} P$ and $X_{e} \in T_{e} G$. From the relation

$$
\kappa_{g} \circ \kappa_{p}=\kappa_{p g} \circ I_{g}^{-1},
$$

where $I_{g}:=L_{g} \circ R_{g}^{-1}$ and $A d_{g}=T I_{g}$, setting $v_{p}=0$ in (9) we obtain the equality

$$
\left(A(p g) \circ T \kappa_{p g}(e) \circ A d_{g^{-1}}(e)\right) X_{e}=\left(T \kappa_{p g}(e) \circ A d_{g^{-1}}(e)\right) X_{e},
$$

valid for any $X_{e} \in T_{e} G$. The above gives (7). In order to show (8) we substitute $X_{e}=0$ into (9) and apply the same arguments as for the previous case.

Now we define the subgroup $A u t_{N} T P \subset A u t_{T G} T P$ consisting of $A \in A u t_{T G} T P$ such that $A(p)=\operatorname{id}_{p}+B(p)$, where $B(p): T_{p} P \rightarrow T_{p}^{v} P$. Conditions (7) and (8) imposed on $A(p)$ written in terms of $B(p)$ take the form

$$
\begin{aligned}
& B(p) \circ T \kappa_{p}(e)=0 \\
& B(p g) \circ T \kappa_{g}(p)=T \kappa_{g}(p) \circ B(p) .
\end{aligned}
$$

From the definition of $B(p)$ and (2) one has

$$
\operatorname{Im} B(p) \subset T_{p}^{v} P \subset \operatorname{Ker} B(p) .
$$

Thus it follows that $B_{1}(p) B_{2}(p)=0$ for any id $+B_{1}$, id $+B_{2} \in A u t_{N} P$. So, one has

$$
A_{1}(p) \circ A_{2}(p)=\left(\operatorname{id}_{p}+B_{1}(p)\right)\left(\operatorname{id}_{p}+B_{2}(p)\right)=\operatorname{id}_{p}+B_{1}(p)+B_{2}(p),
$$

for $A_{1}(p), A_{2}(p) \in A u t_{N} T P$. This shows that $A u t_{N} T P$ is a commutative subgroup of $A u t_{T G} T P$. Therefore, we may identify $A u t_{N} T P$ with the vector subspace $E n d_{N} T P$ of 
EndTP which consists of such endomorphisms $B(p): T_{p} P \rightarrow T_{p} P$ that the property (10) is valid for any $p \in P$.

Now, let us recall that by the definition a connection form on $P$ is a $T_{e} G$-valued differential one-form $\alpha$ satisfying the conditions

$$
\begin{gathered}
\alpha_{p} \circ T \kappa_{p}(e)=\operatorname{id}_{T_{e} G}, \\
\alpha_{p g} \circ T \kappa_{g}(p)=\operatorname{Ad}_{g^{-1}} \circ \alpha_{p}
\end{gathered}
$$

for the value $\alpha_{p}$ of $\alpha$ at $p \in P$ and $g \in G$. Using $\alpha$ one defines the decomposition

$$
T_{p} P=T_{p}^{v} P \oplus T_{p}^{\alpha, h} P
$$

of $T_{p} P$ on the vertical $T_{p}^{v} P$ and the horizontal $T_{p}^{\alpha, h} P:=\operatorname{Ker} \alpha_{p}$ subspaces. Using the decomposition (13) one defines the vector spaces isomorphism

$$
\Gamma_{\alpha}(p): T_{\mu(p)} M \stackrel{\sim}{\rightarrow} T_{p}^{\alpha, h} P
$$

such that

$$
\Gamma_{\alpha}(p g)=T \kappa_{g}(p) \circ \Gamma_{\alpha}(p) \quad \text { and } \quad \operatorname{id}_{T_{\mu(p)} M}=T \mu(p) \circ \Gamma_{\alpha}(p) .
$$

Let us take the decomposition

$$
\operatorname{id}_{T_{p} P}=\Pi_{\alpha}^{v}(p)+\Pi_{\alpha}^{h}(p)
$$

of the identity map of $T_{p} P$ into the sum of projections corresponding to (13). Then we have

$$
\Pi_{\alpha}^{h}(p)=\Gamma_{\alpha}(p) \circ T \mu(p) \text { and } \Pi_{p}^{v}(p)=T \kappa_{p}(e) \circ \alpha_{p} .
$$

Proposition 2. (i) One has the short exact sequence

$$
\left\{\mathrm{id}_{T P}\right\} \rightarrow A t_{N} T P \stackrel{\iota}{\rightarrow} A u t_{T G} T P \stackrel{\lambda}{\rightarrow} A u t_{0} T M \rightarrow\left\{\mathrm{id}_{T M}\right\}
$$

of the group morphisms, where $\iota$ is the inclusion map and $\lambda$ is an epimorphism of Aut $_{T G} T P$ on the group Aut $t_{0} T M$ of the automorphisms of the tangent space TM covering the identity map of $M$ defined by

$$
\left(\lambda(A)(\mu(p))(T \mu(p)) v_{p}\right):=(T \mu(p) \circ A(p)) v_{p},
$$

where $v_{p} \in T_{p} P$.

(ii) Fixing a connection $\alpha$ one defines the injection $\sigma_{\alpha}: A u t_{0} T M \rightarrow A u t_{T G} T P$ by

$$
\sigma_{\alpha}(\tilde{A})(p):=\Pi_{\alpha}^{v}(p)+\Gamma_{\alpha}(p) \circ \tilde{A}(\mu(p)) \circ T \mu(p),
$$

where $\tilde{A} \in A u t_{0} T M$, and the surjection $\beta_{\alpha}: A u t_{T G} T P \rightarrow A u t_{N} T P$ by

$$
\beta_{\alpha}(A):=A \sigma_{\alpha}(\lambda(A))^{-1},
$$


where $A \in A t_{T G} T P$, which are arranged into the short exact sequence

$$
\left\{\operatorname{id}_{T M}\right\} \rightarrow A u t_{0} T M \stackrel{\sigma_{\alpha}}{\longrightarrow} A u t_{T G} T P \stackrel{\beta_{\alpha}}{\longrightarrow} A u t_{N} T P \rightarrow\left\{\operatorname{id}_{T P}\right\},
$$

inverse to the sequence (17), i.e. Im $\sigma_{\alpha}=\beta_{\alpha}^{-1}\left(\mathrm{id}_{T P}\right), \sigma_{\alpha}$ is a right inverse $\pi \circ$ $\sigma_{\alpha}=\mathrm{id}_{T M}$ of $\pi$ and $\beta_{\alpha}$ is the left inverse $\beta_{\alpha} \circ \iota=\mathrm{id}_{T P}$ of $\iota$. The map $\sigma_{\alpha}$ is a monomorphism

$$
\sigma_{\alpha}\left(\tilde{A}_{1} \tilde{A}_{2}\right)=\sigma_{\alpha}\left(\tilde{A}_{1}\right) \sigma_{\alpha}\left(\tilde{A}_{2}\right)
$$

of the groups and $\beta_{\alpha}$ satisfies

$$
\beta_{\alpha}\left(A_{1} A_{2}\right)=\beta_{\alpha}\left(A_{1}\right) \sigma_{\alpha}\left(\lambda\left(A_{1}\right)\right) \beta_{\alpha}\left(A_{2}\right) \sigma_{\alpha}\left(\lambda\left(A_{1}\right)\right)^{-1} .
$$

(iii) The decomposition

$$
A(p)=\left(\operatorname{id}_{p}+B(p)\right) \sigma_{\alpha}(\tilde{A})(p)
$$

of $A \in$ Aut $_{T G} T P$, where $\operatorname{id}_{p}+B(p) \in A u t_{N} T P$ and $\tilde{A} \in A u t_{0} T M$, defines an isomorphism of Aut ${ }_{T G} T P$ with the semidirect product group $A u t_{0} T M \ltimes_{\alpha} E_{n} d_{N} T P$, where the product of $\left(\tilde{A}_{1}, B_{1}\right),\left(\tilde{A}_{2}, B_{2}\right) \in A u t_{0} T M \ltimes_{\alpha} E_{n d} T P$ is given by

$\left[\left(\tilde{A}_{1}, B_{1}\right) \cdot\left(\tilde{A}_{2}, B_{2}\right)\right](p):=\left(\tilde{A}_{1}(\mu(p)) \tilde{A}_{2}(\mu(p)), B_{1}(p)+B_{2}(p) \circ \Gamma_{\alpha}(p) \circ \tilde{A}_{1}^{-1}(\mu(p)) \circ T \mu(p)\right)$.

Proof. From the definition (18) of $\pi$, for all $A_{1}, A_{2} \in A u t_{T G} T P$, we obtain that

$$
\begin{gathered}
\lambda\left(A_{1} A_{2}\right)(\mu(p))\left(T \mu(p) v_{p}\right)=\left(T \mu(p) \circ A_{1}(p)\right)\left(A_{2}(p) v_{p}\right)= \\
=\lambda\left(A_{1}\right)(\mu(p))\left(T \mu(p) \circ A_{2}(p)\right) v_{p}=\left(\lambda\left(A_{1}\right)(\mu(p)) \circ \lambda\left(A_{2}(\mu(p))\right)\left(T \mu(p) v_{p}\right)\right.
\end{gathered}
$$

for $T \mu(p) v_{p} \in T_{\mu(p)} M$. Also, for $\tilde{A}_{1}, \tilde{A}_{2} \in A u t_{0} T M$, from (19) we have

$$
\begin{gathered}
\sigma_{\alpha}\left(\tilde{A}_{1}\right)(p) \circ \sigma_{\alpha}\left(\tilde{A}_{2}\right)(p)= \\
=\left(\Pi_{\alpha}^{v}(p)+\Gamma_{\alpha}(p) \circ \tilde{A}_{1}(\mu(p)) \circ T \mu(p)\right)\left(\Pi_{\alpha}^{v}(p)+\Gamma_{\alpha}(p) \circ \tilde{A}_{2}(\mu(p)) \circ T \mu(p)\right)= \\
=\Pi_{\alpha}^{v}(p)+\Gamma_{\alpha}(p) \circ \tilde{A}_{1}(\mu(p)) \circ T \mu(p) \circ \Gamma_{\alpha}(p) \circ \tilde{A}_{2}(\mu(p)) \circ T \mu(p)= \\
=\Pi_{\alpha}^{v}(p)+\Gamma_{\alpha}(p) \circ \tilde{A}_{1}(\mu(p)) \circ \tilde{A}_{2}(\mu(p)) \circ T \mu(p)=\sigma_{\alpha}\left(\tilde{A}_{1} \tilde{A}_{2}\right)(p) .
\end{gathered}
$$

In order to obtain these equalities we used $\Pi_{\alpha}^{v 2}=\Pi_{\alpha}^{v}$ and $\Pi_{\alpha}^{v} \circ \Gamma_{\alpha}(p)=0$ and $T \mu(p) \circ \Pi_{\alpha}^{v}=$ 0 . Additionally we have the following equalities

$$
\begin{aligned}
& \left(\beta_{\alpha} \circ \iota\right)\left(\mathrm{id}_{T P}+B\right)=\left(\mathrm{id}_{T P}+B\right) \sigma_{\alpha}\left(\mathrm{id}_{T P}\right)=\mathrm{id}_{T P}+B, \\
& \left(\lambda \circ \beta_{\alpha}\right)(A)=\lambda\left(A \sigma_{\alpha}(\lambda(A))^{-1}\right)=\lambda(A) \lambda(A)^{-1}=\mathrm{id}_{T M}
\end{aligned}
$$

and

$$
\left(\lambda \circ \sigma_{\alpha}\right)(\tilde{A})(\mu(p))\left(T \mu(p) v_{p}\right)=\left(\lambda\left(\sigma_{\alpha}(\tilde{A})\right)(\mu(p))\left(T \mu(p) v_{p}\right)=T \mu(p)\left(\sigma_{\alpha}(\tilde{A})(p) v_{p}\right)=\right.
$$




$$
=T \mu(p)\left(\Pi_{\alpha}^{v}(p)+\Gamma_{\alpha}(p) \circ \tilde{A}(\mu(p)) \circ T \mu(p)\right) v_{p}=\tilde{A}(\mu(p))\left(T \mu(p) v_{p}\right) .
$$

Let us also note that $\beta_{\alpha}(A)=\mathrm{id}_{T P}$ if and only if $A=\sigma_{\alpha}(\lambda(A))$. This implies that $\beta_{\alpha}^{-1}\left(\mathrm{id}_{T P}\right)=\operatorname{Im} \sigma_{\alpha}$. Summing up the above statements we prove the points (i) and (ii) of the proposition.

In order to prove (iii) we first note that the decomposition (22) of $A \in A u t_{T G} T P$ into the product of $\operatorname{id}_{T P}+B \in A u t_{N} T P$ and $\sigma_{\alpha}(\tilde{A}) \in \sigma_{\alpha}\left(A u t_{0} T M\right)$ follows from $\lambda \circ \sigma_{\alpha}=\operatorname{id}_{T M}$ and from $A u t_{N} T P=k e r \lambda$. Taking this fact into account one finds

$$
\sigma_{\alpha}(\tilde{A})(p)\left(\operatorname{id}_{p}+B(p)\right) \sigma_{\alpha}\left(\tilde{A}^{-1}\right)(p)=\operatorname{id}_{p}+B(p) \circ \Gamma_{\alpha}(p) \circ \tilde{A}^{-1}(\mu(p)) \circ T \mu(p)
$$

which yields (23).

The structural properties of $A u t_{T G} T P$ described in the above proposition will be useful for the subsequent considerations.

\section{The action of $A u t_{T G} T P$ on the space of connections}

In this section we describe the relationship between the space $\operatorname{Conn} P(M, G)$ of all connections on $P(M, G)$ and the groups from the short exact sequence (17). To this end we define by

$$
\phi_{A}(\alpha)_{p}:=\alpha_{p} \circ A(p)^{-1}
$$

the left action $\phi_{A}: \operatorname{ConnP}(M, G) \rightarrow \operatorname{ConnP}(M, G)$ of $\operatorname{Aut}_{T G} T P$ on $\operatorname{ConnP}(M, G)$, i.e. $\phi$ satisfies $\phi_{A_{1} A_{2}}=\phi_{A_{1}} \circ \phi_{A_{1}}$ for $A_{1}, A_{2} \in A u t_{T G} T P$.

Proposition 3. For the groups $A u t_{T G} T P, A u t_{N} T P$ and $A u t_{0} T M$ one has:

(i) The action of $A_{u t_{T G}} T P$ defined in (24) is transitive.

(ii) The horizontal lift $\Gamma_{\alpha}$ defined by $\alpha \in \operatorname{ConnP}(M, G)$, see (14), satisfies the relation

$$
A(p) \circ \Gamma_{\alpha}(p)=\Gamma_{\phi_{A}(\alpha)}(p) \circ \lambda(A)(\mu(p))
$$

for all $A \in A_{\text {ut }}{ }_{T G} T P$.

(iii) The action (24) restricted to the subgroup $A u t_{N} T P$ is free and transitive.

(iv) The subgroup $\sigma_{\alpha}\left(A u t_{0} T M\right)$ is the stabilizer of $\alpha$ with respect to the action (24).

Proof. (i) In order to show that $\phi_{A}(\alpha) \in \operatorname{Conn} P(M, G)$ we note that it satisfies conditions (11) and (12) if $A$ satisfies (17) and (8). Next let us take the decompositions

$$
\begin{aligned}
& v_{p}=v_{p}^{v}+v_{p}^{h} \\
& v_{p}=v_{p}^{v^{\prime}}+v_{p}^{h^{\prime}}
\end{aligned}
$$

of $v_{p} \in T_{p} P$ on the vertical and horizontal parts with respect to the connections $\alpha$ and $\alpha^{\prime}$, respectively. One easily sees that $A(p): T_{p} P \rightarrow T_{p} P$, defined by

$$
A(p) v_{p}:=v_{p}^{v}+v_{p}^{h^{\prime}}
$$


satisfies conditions (77) and (8) and $\alpha^{\prime}=\phi_{A}(\alpha)$. Thus $A \in A u t_{T G}(T P)$ and the action (24) is transitive.

(ii) The equivariance property (25) follows from $\operatorname{Ker} \phi_{A}\left(\alpha_{p}\right)=A(p) \operatorname{Ker} \alpha_{p}$ and from $T_{p}^{\alpha, h} P=\operatorname{Ker} \alpha_{p}$.

(iii) For any two connections $\alpha, \alpha^{\prime}$ their difference $\alpha-\alpha^{\prime}$ is a $T_{e} G$-valued tensorial one-form, i.e. $\operatorname{Ker}\left(\alpha-\alpha^{\prime}\right)=T_{p}^{v} P$ and $\left(\alpha_{p g}-\alpha_{p g}^{\prime}\right) \circ T \kappa_{g}(p)=A d_{g^{-1}}\left(\alpha_{p}-\alpha_{p}^{\prime}\right)$ for any $p \in P$. Since, for any $p \in P, \alpha$ define the vector space isomorphism $\alpha_{p}: T_{p}^{v} P \rightarrow T_{e} G$ which is the inverse to $T \kappa_{p}(e): T_{e} G \rightarrow T_{p}^{v} P$, see (11), it follows that

$$
B(p):=T \kappa_{p}(e) \circ\left(\alpha_{p}-\alpha_{p}^{\prime}\right)
$$

satisfies (10). From (27) one obtains $\alpha^{\prime}=\phi_{A}(\alpha)$ where $A(p):=\mathrm{id}_{p}+B(p)$. The above proves point (iii).

(iv) Straightforward verification.

The next proposition shows that one can define the subgroup $A u t_{T G} T P \subset A u t_{0} T P$ in terms of the connection space $\operatorname{Conn} P(M, G)$.

Proposition 4. If $A \in A u t_{0}(T P)$ and $\phi_{A}(\operatorname{ConnP}(M, G)) \subset \operatorname{ConnP}(M, G)$ then $A \in$ $A_{\text {ut }}(T P)$.

Proof. Let $A \in \operatorname{Aut}(T P)$ be such that $\phi_{A}(\operatorname{ConnP}(M, G)) \subset \operatorname{ConnP}(M, G)$ then for any $\alpha \in \operatorname{Conn} P(M, G)$ one has

$$
\alpha_{p} \circ A(p) \circ T \kappa_{p}(e)=\alpha_{p} \circ T \kappa_{p}(e)
$$

and

$$
\alpha_{p g} \circ A(p g)^{-1} \circ T \kappa_{g}(e)=\alpha_{p g} \circ T \kappa_{g}(p) \circ A(p)^{-1} .
$$

The above equalities imply (7) and (8) if

$$
\bigcap_{\alpha \in \operatorname{Conn} P(M, G)} \operatorname{Ker} \alpha_{p}=\{0\}
$$

for any $p \in P$. In order to prove (3) we observe that for any vector subspace $H_{p} \subset T_{p} P$ transversal to $T_{p}^{v} P$ and an open subset $\Omega \subset M$ such that $\mu^{-1}(\Omega) \cong G \times \Omega_{p}$ and $\mu(p) \in \Omega$ there exists a local connection form $\alpha$ on $\mu^{-1}(\Omega)$ for which $\operatorname{Ker} \alpha_{p}=H_{p}$. Assuming paracompactness of $M$ and using the decomposition of the unity for the properly chosen covering of $M$ by $\Omega_{p}$ one can construct a connection form $\alpha$ on $M$ such that $H_{p}=$ $\operatorname{Ker} \alpha_{p}$.

Let us note that, given an arbitrary $c \in \mathbb{R} \backslash\{0\}$, any connection $\alpha \in \operatorname{ConnP}(M, G)$ defines a multiplicative one-parameter subgroup of $A u t_{T G} T P$, i.e. $A_{\alpha}^{c_{1}} \circ A_{\alpha}^{c_{2}}=A_{\alpha}^{c_{1} c_{2}}$, for $c_{1}, c_{2} \in \mathbb{R} \backslash\{0\}$ by

$$
A_{\alpha}^{c}(p):=\Pi_{\alpha}^{v}(p)+c \Pi_{\alpha}^{h}(p)=\sigma_{\alpha}\left(c \operatorname{id}_{T M}\right) .
$$




\section{Space of generalized canonical forms on $T^{*} P$}

We recall for further considerations that the standard symplectic form on $T^{*} P$ is $\omega_{0}=d \gamma_{0}$, where $\gamma_{0} \in C^{\infty} T^{*}\left(T^{*} P\right)$ is the canonical one-form on $T^{*} P$ defined at $\varphi \in T^{*} P$ by

$$
\left\langle\gamma_{0 \varphi}, \xi_{\varphi}\right\rangle:=\left\langle\varphi, T \pi^{*}(\varphi) \xi_{\varphi}\right\rangle,
$$

where $\pi^{*}: T^{*} P \rightarrow P$ is the projection of $T^{*} P$ on the base and $\xi_{\varphi} \in T_{\varphi}\left(T^{*} P\right)$.

Let us mention also that by definition a linear vector field on $T^{*} P$ is a pair $(\xi, \chi)$ of vector fields $\xi \in C^{\infty} T\left(T^{*} P\right)$ and $\chi \in C^{\infty} T P$ such that

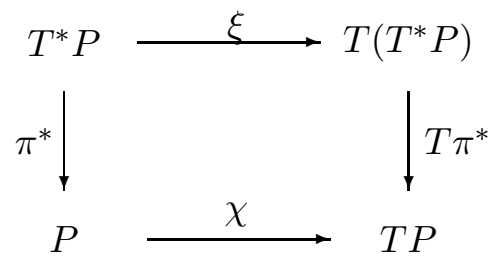

defines a morphism of vector bundles. Note here that $T \pi^{*}(\varphi) \xi_{\varphi}=\chi_{\pi^{*}(\varphi)}$. Regarding the theory of linear vector fields over vector bundles see e.g. Section 3.4 of [5], where their various properties are discussed.

In the sequel we will denote by $\operatorname{Lin} C^{\infty} T\left(T^{*} P\right)$ the Lie algebra of linear vector fields over the vector bundle $\pi^{*}: T^{*} P \rightarrow P$. The Lie bracket of $\left(\xi_{1}, \chi_{1}\right),\left(\xi_{2}, \chi_{2}\right) \in \operatorname{LinC}^{\infty} T\left(T^{*} P\right)$ is defined by

$$
\left[\left(\xi_{1}, \chi_{1}\right),\left(\xi_{2}, \chi_{2}\right)\right]:=\left(\left[\xi_{1}, \xi_{2}\right],\left[\chi_{1}, \chi_{2}\right]\right)
$$

and the vector space structure on $\operatorname{Lin} C^{\infty} T\left(T^{*} P\right)$ by

$$
c_{1}\left(\xi_{1}, \chi_{1}\right)+c_{2}\left(\xi_{2}, \chi_{2}\right):=\left(c_{1} \xi_{1}+c_{2} \xi_{2}, c_{1} \chi_{1}+c_{2} \chi_{2}\right) .
$$

Let $\operatorname{Lin} C^{\infty}\left(T^{*} P\right)$ denote the vector space of smooth fibre-wise linear functions on $T^{*} P$. Notice that spaces $\operatorname{Lin} C^{\infty}\left(T\left(T^{*} P\right)\right)$ and $\operatorname{Lin} C^{\infty}\left(T^{*} P\right)$ have structures of $C^{\infty}(P)$-modules defined by $f(\xi, \chi):=\left(\left(f \circ \pi^{*}\right) \xi, f \chi\right)$ and by $f l:=\left(f \circ \pi^{*}\right) l$, respectively, where $f \in C^{\infty}(P)$ and $l \in \operatorname{LinC}^{\infty}\left(T^{*} P\right)$.

Definition 1. A differential one-form $\gamma \in C^{\infty} T^{*}\left(T^{*} P\right)$ is called a generalized canonical form on $T^{*} P$ if:

(i) $\gamma_{\varphi} \neq 0$ for any $\varphi \in T^{*} P$,

(ii) $\operatorname{kerT} \pi^{*}(\varphi) \subset \operatorname{ker} \gamma_{\varphi}:=\left\{\xi_{\varphi} \in T_{\varphi}\left(T^{*} P\right):\left\langle\gamma_{\varphi}, \xi_{\varphi}\right\rangle=0\right\}$,

(iii) $\langle\gamma, \xi\rangle \in \operatorname{Lin} C^{\infty}\left(T^{*} P\right)$ for any $\xi \in \operatorname{Lin} C^{\infty} T\left(T^{*} P\right)$.

The space of generalized canonical forms on $T^{*} P$ will be denoted by $\operatorname{Can} T^{*} P$. Let us note here that $\gamma_{0} \in \operatorname{CanT}^{*} P$. 
Proposition 5. (i) The map $\Theta: A u t_{0} T P \rightarrow C_{a n T}^{*} P$ defined by

$$
\left.\left\langle\Theta(A)_{\varphi}, \xi_{\varphi}\right\rangle:=\left\langle\varphi, A\left(\pi^{*}(\varphi)\right) T \pi^{*}(\varphi)\right) \xi_{\varphi}\right\rangle,
$$

where $\xi_{\varphi} \in T_{\varphi}\left(T^{*} P\right)$, is bijective.

(ii) The natural left action $L^{*}: A u t_{0} T P \times \operatorname{CanT}^{*} P \rightarrow \operatorname{CanT}^{*} P$ of $A u t_{0} T P$ on $C a n T^{*} P$ defined by

$$
\left\langle\left(L_{A}^{*}(\gamma)\right)_{\varphi}, \xi_{\varphi}\right\rangle:=\left\langle\gamma_{A^{*}(\varphi)}, T A^{*}(\varphi) \xi_{\varphi}\right\rangle,
$$

where $A^{*}: T^{*} P \rightarrow T^{*} P$ is the dual of $A \in A u t_{0} T P$, is a transitive and free action. Furthermore,

$$
L_{A}^{*} \circ \Theta=\Theta \circ L_{A},
$$

where $L_{A} A^{\prime}:=A A^{\prime}$, i.e. $L_{A}^{*} \Theta\left(A^{\prime}\right)=\Theta\left(A A^{\prime}\right)$.

Proof. (i) If $\Theta\left(A_{1}\right)=\Theta\left(A_{2}\right)$, then using (29), for any $T \pi^{*}(\varphi) \xi_{\varphi} \in T_{\pi^{*}(\varphi)} P$ we obtain

$$
\left\langle\left(A_{1}\left(\pi^{*}(\varphi)\right)^{*}-A_{2}\left(\pi^{*}(\varphi)\right)^{*}\right) \varphi, T \pi^{*}(\varphi) \xi_{\varphi}\right\rangle=0 .
$$

This gives $A_{1}=A_{2}$. So, $\Theta$ is an injection.

Let us take $\gamma \in \operatorname{CanT}^{*} P$. Then $\langle\gamma, \xi\rangle \in \operatorname{Lin} C^{\infty}\left(T^{*} P\right)$ if $\langle\xi, \chi\rangle \in \operatorname{Lin}^{\infty}\left(T\left(T^{*} P\right)\right)$. By virtue of the point (ii) of Definition 1 the fibre-wise linear functions $\langle\gamma, \xi\rangle$ depend only on vector fields $\chi=T \pi^{*} \xi \in C^{\infty} T P$ and this dependence defines a morphism of $C^{\infty}(P)$ modules. On the other hand one can consider $\langle\gamma, \xi\rangle$ as a section of $T^{* *} P \cong T P$. Thus we can represent it as

$$
\langle\gamma, \xi\rangle(\varphi)=\left\langle\varphi, \chi^{\prime}\left(\pi^{*}(\varphi)\right\rangle\right.
$$

by some vector field $\chi^{\prime} \in C^{\infty} T P$. The dependence between $\langle\gamma, \xi\rangle$ and $\chi^{\prime}$ given by (31) is also a morphism of $C^{\infty}(P)$-modules. Therefore, there exists $A \in E n d_{0} T P$ such that

$$
\chi^{\prime}(p)=A(p) \chi(p)
$$

and we have $\gamma=\Theta(A)$. Substituting (32) into (31) we obtain

$$
\left\langle\gamma_{\varphi}, \xi_{\varphi}\right\rangle=\left\langle\varphi, A\left(\pi^{*}(\varphi)\right) T \pi^{*}(\varphi) \xi_{\varphi}\right\rangle=\left\langle A\left(\pi^{*}(\varphi)\right)^{*} \varphi, T \pi^{*}(\varphi) \xi_{\varphi}\right\rangle
$$

and, thus $\gamma=\Theta(A)$.

Let us assume that $A \notin A u t_{0} T P$. Then there exists $\varphi$ such that $A\left(\pi^{*}(\varphi)\right)^{*} \varphi=0$. From (34) we see that for this $\varphi$ we have $\gamma_{\varphi}=0$, which contradicts the point (i) of Definition 1. So, $A \in A u t_{0} T P$ and thus $\Theta$ is a surjection. The above proves (i).

(ii) Since any element of $\operatorname{CanT}^{*} P$ can be written as $\Theta\left(A^{\prime}\right)$ for some $A^{\prime} \in A u t_{0} T P$ we obtain from the definition (29) that

$$
\begin{gathered}
\left\langle L_{A}^{*}\left(\Theta\left(A^{\prime}\right)\right)_{\varphi}, \xi_{\varphi}\right\rangle=\left\langle\Theta\left(A^{\prime}\right)_{A^{*}(\varphi)}, T A^{*}(\varphi) \xi_{\varphi}\right\rangle=\left\langle A^{*}(\varphi), A^{\prime}\left(\pi^{*}(\varphi)\right) \circ T \pi^{*}(\varphi) \circ T A^{*}(\varphi) \xi_{\varphi}\right\rangle= \\
=\left\langle\varphi, A\left(\pi^{*}(\varphi)\right) \circ A^{\prime}\left(\pi^{*}(\varphi)\right) \circ T\left(\pi^{*} \circ A^{*}\right)(\varphi) \xi_{\varphi}\right\rangle=\left\langle\varphi, A\left(\pi^{*}(\varphi)\right) \circ A^{\prime}\left(\pi^{*}(\varphi)\right) \circ T\left(\pi^{*}\right)(\varphi) \xi_{\varphi}\right\rangle= \\
=\left\langle\Theta\left(A A^{\prime}\right)_{\varphi}, \xi_{\varphi}\right\rangle,
\end{gathered}
$$

which proves (30). From (30) and from the point (i) of the proposition it follows that $L^{*}$ is a transitive and free action. 
From the above proposition we conclude that $\gamma \in \operatorname{CanT}^{*} P$ is the pull-back $\gamma=\Theta(A)=$ $L_{A}^{*} \gamma_{0}$ of the canonical form $\gamma_{0}$. So, $\omega_{A}:=d \Theta(A)$ is a symplectic form.

The lift $\Phi_{g}^{*}: T^{*} P \rightarrow T^{*} P$ of the action $\kappa_{g}: P \rightarrow P$ to the cotangent bundle $T^{*} P$ is defined by

$$
\Phi_{g}^{*}(\varphi)(p g)=\left(T \kappa_{g}(p)^{-1}\right)^{*} \varphi
$$

where $p=\pi^{*}(\varphi)$.

If $\gamma \in \operatorname{CanT}^{*} P$ is $G$-invariant with respect to (33), then $\mathcal{L}_{\xi^{x}} \gamma=0$ for $X \in T_{e} G$, where $\xi^{X} \in C^{\infty} T\left(T^{*} P\right)$ is the fundamental vector field, i.e. the vector field tangent to the flow $t \rightarrow \Phi_{\exp t x}^{*}$. So, for a $G$-invariant symplectic form $\omega_{A}=d \gamma=d \Theta(A)$ one has

$$
\xi^{X}\left\llcorner\omega=-d\left\langle J_{A}, X\right\rangle\right.
$$

where the $G$-equivariant momentum map $J_{A}: T^{*} P \rightarrow T_{e}^{*} G$ is given by $J_{A}=J_{0} \circ A^{*}$. We note here that for the standard symplectic form $\omega_{0}=d \gamma_{0}$ the momentum map is

$$
J_{0}(\varphi)=\varphi \circ T \kappa_{\pi^{*}(\varphi)}(e)
$$

It is reasonable to define the space

$$
\operatorname{Can}_{T G} T^{*} P:=\Theta\left(\text { Aut }_{T G} T P\right)
$$

which is an $A u t_{T G} T P$-invariant subspace of the space $\operatorname{CanT}^{*} P$.

Proposition 6. (i) The generalized canonical form $\Theta(A)$ belongs to $C a n_{T G} T^{*} P$ if and only if $\left(\phi_{g}^{*}\right)^{*} \Theta(A)=\Theta(A)$ and $J_{A}=J_{0}$.

(ii) One can consider $\operatorname{Can}_{T G} T^{*} P$ as the orbit of the subgroup $A u t_{T G} T P \subset$ Aut $_{0} T P$ taken through $\gamma_{0}$ with respect to the free action $L^{*}$ defined in (29).

(iii) If $A \in A u t_{0} T P$ and $L_{A}^{*}\left(\operatorname{Can}_{T G} T^{*} P\right) \subset \operatorname{Can}_{T G} T^{*} P$ then $A \in A_{4} t_{T G} T P$.

Proof. (i) The canonical form $\Theta(A)$ is $G$-invariant if and only if

$$
\left\langle\Theta(A)_{\Phi_{g}^{*}(\varphi)}, T \Phi_{g}^{*}(\varphi) \xi_{\varphi}\right\rangle=\left\langle\Theta(A)_{\varphi}, \xi_{\varphi}\right\rangle
$$

for any $g \in G$. For the left hand side of (34) we have

$$
\begin{gathered}
\left\langle\left(\phi_{g}^{*}\right)^{*} \Theta(A)_{\varphi}, \xi_{\varphi}\right\rangle=\left\langle\Theta(A)_{\Phi_{g}^{*}(\varphi)}, T \Phi_{g}^{*}(\varphi) \xi_{\varphi}\right\rangle= \\
=\left\langle\Phi_{g}^{*}(\varphi), A\left(\pi^{*}\left(\Phi_{g}^{*}(\varphi)\right) T \pi^{*}\left(\Phi_{g}^{*}(\varphi)\right) T \Phi_{g}^{*}(\varphi) \xi_{\varphi}\right\rangle=\right. \\
=\left\langle\varphi, T \kappa_{g}(p)^{-1} A\left(\pi^{*} \circ \Phi_{g}^{*}\right)(\varphi) T\left(\pi^{*} \circ \Phi_{g}^{*}\right)(\varphi) \xi_{\varphi}\right\rangle= \\
=\left\langle\varphi, T \kappa_{g}(p)^{-1} A\left(\pi^{*}(\varphi) g\right) T \kappa_{g}\left(\pi^{*}(\varphi)\right) T \pi^{*}(\varphi) \xi_{\varphi}\right\rangle=\left\langle\Theta(A)_{\varphi}, \xi_{\varphi}\right\rangle
\end{gathered}
$$

for any $\varphi \in T^{*} P$ and $\xi_{\varphi} \in T_{\varphi}\left(T^{*} P\right)$. Note that the second equality in (35) follows from $\pi^{*} \circ \Phi_{g}^{*}=\kappa_{g} \circ \pi^{*}$. From (35) and from the definition (28) we obtain the condition (8). 
From $J_{A}=J_{0}$ we have

$$
\begin{gathered}
\langle J(\varphi), X\rangle=\left\langle\Theta(A)_{\varphi}, \xi_{\varphi}^{X}\right\rangle=\left\langle\varphi, A\left(\pi^{*}(\varphi)\right) T \kappa_{\pi^{*}(\varphi)}(e) X\right\rangle= \\
=\left\langle\varphi, T \kappa_{\pi^{*}(\varphi)}(e) X\right\rangle
\end{gathered}
$$

for all $\varphi \in T^{*} P$ and $X \in T_{e} G$. This shows that an element $A \in A u t_{0} T P$ satisfies (7).

(ii) This statement follows from (i) and from $L_{A}^{*} \gamma_{0}=\Theta(A)$.

(iii) If $L_{A}^{*} \operatorname{Can}_{T G} T^{*} P \subset \operatorname{Can}_{T G} T^{*} P$ then $L_{A}^{*} \Theta\left(A_{1}\right)=\Theta\left(A A_{1}\right) \in \operatorname{Can}_{T G} T^{*} P$ for any $A_{1} \in A u t_{T G} T P$. So, due to the property (i) we have $A A_{1} \in A u t_{T G} T P$ and, thus $A \in A u t_{T G} T P$.

In Section 2 we fixed a reference connection $\alpha$ in order to investigate the structure of group $A u t_{T G} T P$, see Proposition 2, Now, taking into consideration Proposition 6 we study the structure of generalized canonical forms $\Theta(A) \in \operatorname{Can}_{T G} T^{*} P$ using decompositions (22) and (15), and (16). We obtain

$$
\begin{aligned}
& A(p)=\Pi_{\alpha}^{v}(p)+\Pi_{\alpha}^{v}(p) A(p) \Pi_{\alpha}^{h}(p)+\Pi_{\alpha}^{h}(p) A(p) \Pi_{\alpha}^{h}(p)= \\
= & T \kappa_{p}(e) \circ \alpha_{p}+\left(\operatorname{id}_{T P}+B\right)(p) \circ \Gamma_{\alpha}(p) \circ \tilde{A}(\mu(p)) \circ T \mu(p),
\end{aligned}
$$

where $\operatorname{id}_{T P}+B \in A u t_{N} T P$ and $\tilde{A} \in A u t_{0} T M$. Substituting $A$ given by (36) into the definition (28) we find the corresponding formula for $\Theta(A)$

$$
\begin{gathered}
\Theta(A)(\varphi)=\varphi \circ T \kappa_{\pi^{*}(\varphi)} \circ \alpha_{\pi^{*}(\varphi)}+ \\
+\varphi \circ\left(\operatorname{id}_{T P}+B\right)\left(\pi^{*}(\varphi)\right) \circ \Gamma_{\alpha}\left(\pi^{*}(\varphi)\right) \circ \tilde{A}\left(\left(\mu \circ \pi^{*}\right)(\varphi)\right) \circ T\left(\mu \circ \pi^{*}\right)(\varphi) .
\end{gathered}
$$

In particular cases when $\tilde{A}=\operatorname{id}_{T M}$ and $B=0$ we have

$$
\begin{aligned}
& \Theta(\mathrm{id}+B)(\varphi)=\varphi \circ T \pi^{*}(\varphi)+\varphi \circ B\left(\pi^{*}(\varphi)\right) \circ T \pi^{*}(\varphi)= \\
= & \varphi \circ T \pi^{*}(\varphi)+\varphi \circ T \kappa_{\pi^{*}(\varphi)}(e) \circ\left(\alpha_{\pi^{*}(\varphi)}^{\prime}-\alpha_{\pi^{*}(\varphi)}\right) \circ T \pi^{*}(\varphi)
\end{aligned}
$$

and

$$
\Theta\left(\sigma_{\alpha}(\tilde{A})\right)(\varphi)=J_{0}(\varphi) \circ \alpha_{\pi^{*}(\varphi)} \circ T \pi^{*}(\varphi)+\varphi \circ \Gamma_{\alpha}\left(\pi^{*}(\varphi)\right) \circ \tilde{A}\left(\left(\mu \circ \pi^{*}\right)(\varphi)\right) \circ T\left(\mu \circ \pi^{*}\right)(\varphi),
$$

respectively. Let us note that $\Theta\left(\sigma_{\alpha}\left(\operatorname{id}_{T M}\right)\right)=\Theta\left(\operatorname{id}_{T P}\right)=\varphi \circ T \pi^{*}(\varphi)$, i.e. $\Theta\left(\sigma_{\alpha}\left(\operatorname{id}_{T M}\right)\right)$ is the canonical one-form $\gamma_{0}$.

Corrolary 7. Fixing a connection $\alpha$ one obtains from (38) an embedding $\iota_{\alpha}: \operatorname{ConnP}(M, G) \hookrightarrow$ $\operatorname{Can}_{T G} T^{*} P$ of the connection space into the space of generalized canonical forms defined as follows

$$
\iota_{\alpha}\left(\alpha^{\prime}\right):=\varphi \circ T \pi^{*}(\varphi)+\varphi \circ T \kappa_{\pi^{*}(\varphi)}(e) \circ\left(\alpha_{\pi^{*}(\varphi)}^{\prime}-\alpha_{\pi^{*}(\varphi)}\right) \circ T \pi^{*}(\varphi) .
$$

The symplectic form $d \iota_{\alpha}\left(\alpha^{\prime}\right)$ is the pullback $L_{\mathrm{id}_{T P}+B} \omega_{0}$ of the standard symplectic form $\omega_{0}$ by the bundle morphism $\left(\operatorname{id}_{T P}+B\right)^{*}: T^{*} P \rightarrow T^{*} P$, where $\operatorname{id}_{T P}+B \in A u t_{N} T P$ is defined in (27). 
Although there is no distinguished connection on a principal $G$-bundle in general, such connections exist in some particular cases. For example, if the principal bundle $P(M, G)$ is trivial, $P=M \times G$, or when $P$ is a Lie group and $G$ is its subgroup. In the last case there exists the connection which is invariant with respect to the left action of $P$ on itself and this connection is defined in a unique way, see Theorem 11.1 in [3]. Let us mention that in the case when the reference connection $\alpha$ is determined by some additional conditions, for example by the symmetry properties, then the connection $\alpha^{\prime}$, see (39), can be naturally interpreted as an external field which interacts with a particle localized in the configuration space $P$.

In [7, 11] a $G$-equivariant diffeomorphism $I_{\alpha}: T^{*} P \stackrel{\sim}{\rightarrow} \bar{P} \times T_{e}^{*} G$ dependent on a fixed connection $\alpha$ was considered, where

$$
\bar{P}:=\left\{(\tilde{\varphi}, p) \in T^{*} M \times P: \tilde{\pi}^{*}(\tilde{\varphi})=\mu(p)\right\}
$$

is the total space of the principal bundle $\bar{P}\left(T^{*} M, G\right)$ being the pullback of the principalbundle $P(M, G)$ to $T^{*} M$ by the projection $\tilde{\pi}^{*}: T^{*} M \rightarrow M$ of $T^{*} M$ on the base $M$. This diffeomorphism is defined as follows

$$
I_{\alpha}(\varphi):=\left(\Gamma_{\alpha}^{*}\left(\pi^{*}(\varphi)\right)(\varphi), \pi^{*}(\varphi), J_{0}(\varphi)\right) .
$$

The correctness of the above definition follows from $\tilde{\pi}^{*} \circ \Gamma_{\alpha}^{*}=\mu \circ \pi^{*}$. The map $I_{\alpha}^{-1}$ : $\bar{P} \times T_{e}^{*} G \rightarrow T^{*} P$ given by

$$
I_{\alpha}^{-1}(\tilde{\varphi}, p, \chi)=\tilde{\varphi} \circ T \mu(p)+\chi \circ \alpha_{p}
$$

is the inverse to $I_{\alpha}$.

The natural right action of $A u t_{T G} T P$ on $T^{*} P$, defined for $A \in A u t_{T G} T P$ by $\left(A^{*} \varphi\right)\left(\pi^{*}(\varphi)\right):=$ $\varphi \circ A\left(\pi^{*}(\varphi)\right)$, and the action of $G$ on $T^{*} P$ defined in (33) transported by $I_{\alpha}$ to $\bar{P} \times T_{e}^{*} G$ are given by

$$
\begin{aligned}
& \Lambda_{\alpha}(A)(\tilde{\varphi}, p, \chi):=\left(I_{\alpha} \circ A^{*} \circ I_{\alpha}^{-1}\right)(\tilde{\varphi}, p, \chi)= \\
& =\left(\left(\tilde{\varphi} \circ T \mu(p)+\chi \circ \alpha_{p}\right) \circ A(p) \circ \Gamma_{\alpha}(p), p, \chi\right)
\end{aligned}
$$

and by

$$
\psi_{g}^{*}(\tilde{\varphi}, p, \chi):=\left(I_{\alpha} \circ \phi_{g}^{*} \circ I_{\alpha}^{-1}\right)(\tilde{\varphi}, p, \chi)=\left(\tilde{\varphi}, p g, A d_{g^{-1}}^{*} \chi\right),
$$

respectively. Setting $A=\operatorname{id}_{T P}+B$ or $A=\sigma_{\alpha}(\tilde{A})$ in (41) we obtain

$$
\Lambda_{\alpha}\left(\operatorname{id}_{T P}+B\right)(\tilde{\varphi}, p, \chi)=\left(\tilde{\varphi}+\chi \circ \alpha_{p} \circ B(p) \circ \Gamma_{\alpha}(p), p, \chi\right)
$$

or

$$
\Lambda_{\alpha}\left(\sigma_{\alpha}(\tilde{A})\right)(\tilde{\varphi}, p, \chi)=(\tilde{\varphi} \circ \tilde{A}, p, \chi),
$$

respectively. Summarizing, let us mention some properties of the above two actions:

(i) The action $\Lambda_{\alpha}$ of $A u t_{T G} T P$ on $\bar{P} \times T_{e}^{*} G$ is reduced to an action of $A u t_{T G} T P$ on $T^{*} M$ which preserves the cotangent spaces $T_{m}^{*} M, m \in M$, and is realized on them by affine maps, see (41), (43) and (44). 
(ii) The action (42) of $G$ does not change $\tilde{\varphi}$ and commute with the action (41) of the group $A_{\text {ut }} T P$.

Using $I_{\alpha}^{-1}: \bar{P} \times T_{e}^{*} G \rightarrow T^{*} P$ we pull the generalized canonical form $\Theta(A)$ back to $\bar{P} \times T_{e}^{*} G$. For this reason note that a vector $\xi_{(\tilde{\varphi}, p, \chi)} \in T_{(\tilde{\varphi}, p, \chi)}\left(T^{*} M \times P \times T_{e}^{*} G\right)$ is tangent to $\bar{P} \times T_{e}^{*} G \subset T^{*} M \times P \times T_{e}^{*} G$ if and only if

$$
T\left(\tilde{\pi}^{*} \circ p r_{1}\right)(\tilde{\varphi}, p, \chi) \xi_{(\tilde{\varphi}, p, \chi)}=T\left(\mu \circ p r_{2}\right)(\tilde{\varphi}, p, \chi) \xi_{(\tilde{\varphi}, p, \chi)},
$$

where $\operatorname{pr}_{1}(\tilde{\varphi}, p, \chi):=\tilde{\varphi}$ and $p r_{2}(\tilde{\varphi}, p, \chi):=p$. The equality (45) follows from $\tilde{\pi}^{*} \circ p r_{1}=$ $\mu \circ p r_{2}$. For $A=\left(\operatorname{id}_{T P}+B\right) \sigma_{\alpha}(\tilde{A})$ we have

$$
\begin{gathered}
\left\langle\left(I_{\alpha}^{-1}\right)^{*} \Theta(A)(\tilde{\varphi}, p, \chi), \xi_{(\tilde{\varphi}, p, \chi)}\right\rangle= \\
=\left\langle I_{\alpha}^{-1}(\tilde{\varphi}, p, \chi), A\left(\pi^{*}\left(I_{\alpha}^{-1}(\tilde{\varphi}, p, \chi)\right)\right) \circ T \pi^{*}\left(I_{\alpha}^{-1}(\tilde{\varphi}, p, \chi)\right) \circ T I_{\alpha}^{-1}(\tilde{\varphi}, p, \chi) \xi_{(\tilde{\varphi}, p, \chi)}\right\rangle= \\
=\left\langle I_{\alpha}^{-1}(\tilde{\varphi}, p, \chi), A\left(\pi^{*} \circ I_{\alpha}^{-1}\right)(\tilde{\varphi}, p, \chi) \circ T\left(\pi^{*} \circ I_{\alpha}^{-1}\right)(\tilde{\varphi}, p, \chi) \xi_{(\tilde{\varphi}, p, \chi)}\right\rangle= \\
=\left\langle\tilde{\varphi} \circ T \mu(p)+\chi \circ \alpha_{p}, A(p) T p r_{2}(\tilde{\varphi}, p, \chi) \xi_{(\tilde{\varphi}, p, \chi)}\right\rangle= \\
=\left\langle\tilde{\varphi} \circ \tilde{A}(\mu(p)), T \mu(p) \circ T p r_{2}(\tilde{\varphi}, p, \chi) \xi_{(\tilde{\varphi}, p, \chi)}\right\rangle+\left\langle\chi \circ \alpha_{p}, A(p) \operatorname{Tpr}_{2}(\tilde{\varphi}, p, \chi) \xi_{(\tilde{\varphi}, p, \chi)}\right\rangle= \\
=\left\langle\tilde{\varphi} \circ \tilde{A}(\mu(p)), T\left(\tilde{\pi}^{*} \circ p r_{1}\right)(\tilde{\varphi}, p, \chi) \xi_{(\tilde{\varphi}, p, \chi)}\right\rangle+\left\langle\chi \circ \alpha_{p}, A(p) \circ \operatorname{Tpr}_{2}(\tilde{\varphi}, p, \chi) \xi_{(\tilde{\varphi}, p, \chi)}\right\rangle,
\end{gathered}
$$

where we have used (40), (45) and $\pi^{*} \circ I_{\alpha}^{-1}=p r_{2}$. Omitting $\xi_{(\tilde{\varphi}, p, \chi)}$ in (46) we obtain

$$
\begin{gathered}
\left(I_{\alpha}^{-1}\right)^{*} \Theta(A)(\tilde{\varphi}, p, \chi)= \\
=\tilde{\varphi} \circ \tilde{A}(\mu(p)) \circ T\left(\tilde{\pi}^{*} \circ p r_{1}\right)(\tilde{\varphi}, p, \chi)+\chi \circ \alpha_{p} \circ A(p) \circ \operatorname{Tpr}_{2}(\tilde{\varphi}, p, \chi)= \\
=p r_{1}^{*}\left(\tilde{\Theta}(\tilde{A})(\tilde{\varphi}, p, \chi)+\left\langle p r_{3}(\tilde{\varphi}, p, \chi), p r_{2}^{*}\left(\Phi_{A^{-1}}(\alpha)\right)(\tilde{\varphi}, p, \chi)\right\rangle,\right.
\end{gathered}
$$

where $\operatorname{pr}_{3}(\tilde{\varphi}, p, \chi):=\chi$. The symplectic form corresponding to (47) is given by

$$
\begin{gathered}
d\left(\left(I_{\alpha}^{-1}\right)^{*} \Theta(A)\right)= \\
=p r_{1}^{*}(d \tilde{\Theta}(\tilde{A}))+\left\langle d p r_{3} \stackrel{\wedge}{\wedge} r_{2}^{*}\left(\Phi_{A^{-1}}(\alpha)\right)\right\rangle+\left\langle p r_{3}, p r_{2}^{*}\left(d \Phi_{A^{-1}}(\alpha)\right)\right\rangle .
\end{gathered}
$$

Let us note that $\left(I_{\alpha}^{-1}\right)^{*} \Theta(A)$ consists of the pull back on $\bar{P} \times T_{e}^{*} G$ of the generalized canonical form $\tilde{\Theta}(\tilde{A}) \in \operatorname{CanT}^{*} M$ by $p r_{1}: \bar{P} \times T_{e}^{*} G \rightarrow T^{*} M$ and the part defined by the connection form $\Phi_{A^{-1}}(\alpha)$. 


\section{The Marsden-Weinstein reduction}

Considering $P$ as the configuration space of a physical system which has a symmetry described by $G$ one consequently assumes that its Hamiltonian $H \in C^{\infty}\left(T^{*} P\right)$ is a $G$ invariant function on $T^{*} P$, i.e. $H \circ \phi_{g}^{*}=H$ for $g \in G$. Hence it is natural to consider the class of Hamiltonian systems on $G$-symplectic manifold $\left(T^{*} P, \omega_{A}, J_{0}\right)$ with a $G$-invariant Hamiltonians $H$.

Using the isomorphism $\left(T^{*} P, \omega_{A}, J_{0}\right) \cong\left(\bar{P} \times T_{e}^{*} G,\left(I_{\alpha}^{-1}\right)^{*} \omega_{A}, p r_{3}\right)$ of $G$-symplectic manifolds, where the symplectic form $\left(I_{\alpha}^{-1}\right)^{*} \omega_{A}$ is presented in (48) and the momentum map is $J_{0} \circ I_{\alpha}=p r_{3}$, one defines (see [7, 11]) the $G$-invariant Hamiltonian $H \in C^{\infty}\left(\bar{P} \times T_{e}^{*} G\right)$ as follows

$$
H(\tilde{\varphi}, p, \chi):=(\tilde{H} \circ \bar{\mu})(\tilde{\varphi}, p, \chi)+\left(C \circ p r_{3}\right)(\tilde{\varphi}, p, \chi),
$$

where $\bar{\mu}: \bar{P} \rightarrow T^{*} M$ is the projection of the total space $\bar{P}$ of the principal $G$-bundle $\bar{P}\left(T^{*} M, G\right)$ on the base $T^{*} M$ and $\tilde{H} \in C^{\infty}\left(T^{*} M\right)$. Coming back to the phase space $\left(T^{*} P, \omega_{A}, J_{0}\right)$ one obtains the $G$-Hamiltonian system with the Hamiltonian

$$
H_{\alpha}(\varphi):=\left(H \circ I_{\alpha}\right)(\varphi)=\left(\tilde{H} \circ \Gamma_{\alpha}^{*}\right)(\varphi)+\left(C \circ J_{0}\right)(\varphi) .
$$

Let us stress that in the case of $\left(T^{*} P, \omega_{A}, J_{0}, H_{\alpha}\right)$ only the Hamiltonian $H_{\alpha}$ of the system depends on $\alpha \in \operatorname{ConnP}(M, G)$ and in the case of $\left(\bar{P} \times T_{e}^{*} G,\left(I_{\alpha}^{-1}\right)^{*} \omega_{A}, p r_{3}, H\right)$ the only symplectic form $\left(I_{\alpha}^{-1}\right)^{*} \omega_{A}$.

In $[2,4,7,8,9,10,11,12,13$, there were presented various models of the description of motion of a classical particle in the external Yang-Mills field given by the connection $\alpha$ and by the Hamiltonian $H$. In these models the basic symplectic structure on $T^{*} P$ is given by the standard symplectic form $\omega_{0}$. Here allowing the generalized symplectic form $\omega_{A}$ we extend the class of models under investigations.

The $G$-invariance of the Hamiltonian system $\left(T^{*} P, \omega_{A}, J_{0}, H_{\alpha}\right)$ allows ones to apply the Marsden-Weinstein reduction procedure [6]. For this reason we consider the dual pair of Poisson manifolds

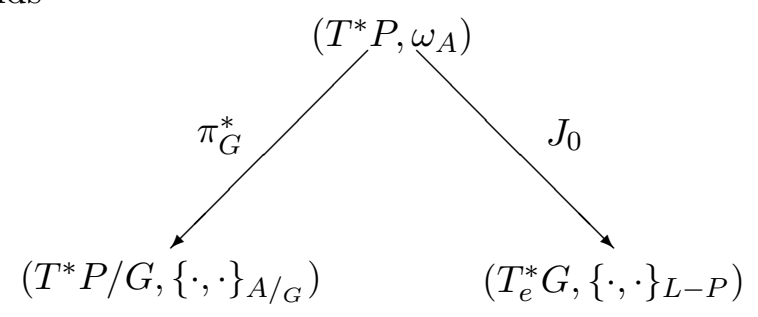

in the sense of Subsection 9.3 in [1]. Recall that the symplectic form $\omega_{A}$ is a $G$-invariant two-form. The Poisson bracket $\{f, g\}_{A / G}$ of $f, g \in C^{\infty}\left(T^{*} P / G\right)$ is defined by $\left\{f \circ \pi_{G}^{*}, g \circ\right.$ $\left.\pi_{G}^{*}\right\}_{A}$, where we identify $C^{\infty}\left(T^{*} P / G\right)$ with the Poisson subalgebra $C_{G}^{\infty}\left(T^{*} P\right) \subset C^{\infty}\left(T^{*} P\right)$ of $G$-invariant functions and $\{\cdot, \cdot\}_{A}$ is the Poisson bracket on $C^{\infty}\left(T^{*} P\right)$ defined by $\omega_{A}$. By $\{\cdot, \cdot\}_{L-P}$ we denoted Lie-Poisson bracket on the dual $T_{e}^{*} G$ of the Lie algebra $T_{e} G$.

Note that surjective submersions in (49) are Poisson maps and the Poisson subalgebras $\left(\pi_{G}^{*}\right)^{*}\left(C^{\infty}\left(T^{*} P / G\right)\right)$ and $J_{0}^{*}\left(C^{\infty}\left(T_{e}^{*} G\right)\right)$ are mutually polar. As a consequence of the above one obtains the one-to-one correspondence between the coadjoint orbits $\mathcal{O} \subset T_{e}^{*} G$ of $G$ 
and the symplectic leaves $\mathcal{S} \subset T^{*} P / G$ of the Poisson manifold $\left(T^{*} P / G,\{\cdot, \cdot\}_{A / G}\right)$ which is defined as follows

$$
\mathcal{S}=\pi_{G}^{*}\left(J_{0}^{-1}(\mathcal{O})\right) \quad \text { and } \quad \mathcal{O}=J_{0}\left(\pi_{G}^{*-1}(\mathcal{S})\right) .
$$

Let us stress that the manifold structure of a symplectic leaf $\mathcal{S}$ does not depend on the choise of $A \in A u t_{T G} T P$, but its symplectic structure $\omega_{A}^{\mathcal{S}}$ does. The action of $A u t_{T G} T P$ on $T^{*} P$ commutes with the action (33) of $G$ on $T^{*} P$, so, it defines an action of $A u t_{T G} T P$ on the quotient manifold $T^{*} P / G$. By the definition of $\operatorname{Can}_{T G} T^{*} P$, the momentum map $J_{A}$ for $\omega_{A}$ coincides with $J_{0}=J_{A}$. Thus we conclude that the action of $A^{\prime} \in A u t_{T G} T P$ on $T^{*} P / G$ preserves the symplectic leaves $\mathcal{S}$ and transforms their symplectic forms in the following way $\omega_{A}^{\mathcal{S}} \rightarrow \omega_{A^{\prime} A}^{\mathcal{S}}$.

Since $I_{\alpha}: T^{*} P \rightarrow \stackrel{M}{P} \times T_{e}^{*} G$ is a $G$-equivariant map it defines a diffeomorphism

$$
\left[I_{\alpha}\right]: T^{*} P / G \rightarrow \bar{P} \times_{A d_{G}^{*}} T_{e}^{*} G
$$

of the quotient manifolds which transports the Poisson structure $\{\cdot, \cdot\}_{A / G}$ of $T^{*} P / G$ on the total space $\bar{P} \times_{A d_{G}^{*}} T_{e}^{*} G$ of the vector bundle $\bar{P} \times_{A d_{G}^{*}} T_{e}^{*} G \rightarrow T^{*} M$ over the symplectic manifold $\left(T^{*} M, d \tilde{\Theta}(\tilde{A})\right)$. Using (15) one obtains the isomorphisms $\left[I_{\alpha, \mathcal{O}}\right]=\pi_{G}^{*}\left(J_{0}^{-1}(\mathcal{O})\right) \stackrel{\sim}{\rightarrow}$ $\bar{P} \times A d_{G}^{*} \mathcal{O}$ of symplectic leaves. If $A=\sigma_{\alpha}\left(\tilde{\mathrm{id}}_{T M}\right)=\mathrm{id}_{T P}$ one obtains the diffeomorphisms of symplectic leaves constructed in [7, 11] where the coadjoint orbit $\mathcal{O}$ is the phase space for inner degrees of freedom. In this case the symplectic manifold $\left(T^{*} M, d \tilde{\gamma}_{0}\right)$ is the phase space for external degrees of freedom and $\bar{P} \times_{A d_{G}^{*}} \mathcal{O}$ is the total phase space of a classical particle interacted with Yang-Mills field described by $\alpha$ which was constructed in [11].

If $\rho \in T_{e}^{*} G$ is such that $A d_{G}^{*} \rho=\rho$ then $\mathcal{O}=\{\rho\}$. Hence the symplectic leaf $\mathcal{S}=$ $\bar{P} \times A d_{G}^{*} \mathcal{O}$ is isomorphic as a manifold with $T^{*} M$ but the reduced symplectic form $\omega_{A}^{S}$ of $\mathcal{S}$ depends on the choice of $A \in A u t_{T G} T P$. For example the above situation happens for all $\rho \in T_{e}^{*} G$ if $G$ is a commutative group or if $\rho=0$.

Ending let us mention that all constructions presented above have an equivariance properties with respect to the group $A u t_{T G} T P$.

\section{References}

[1] Coste, A., Dazord, P., Weinstein, A.: Groupoïdes symplectiques, Publications du Département de Mathématiques de l'Université Lyon, Dept. Math. Univ. ClaudeBernard Lyon I, 1-67 (1987)

[2] B. Kerner.. Generalization of the Kaluza-Klein theory for an arbitrary non-abelian gauge groupoid, Ann.Inst.Henri Poincare. 9. 143-152 (1968)

[3] S. Kobayashi, K. Nomizu. Foundations of Differential Geometry, volume 1 Interscience Publishers, 1963.

[4] M.Kummer. On the construction of the reduced phase space of a Hamiltonian system with symmetry, Indiana University Math, 30, 281-291 (1981) 
[5] K. C. H. Mackenzie. General theory of Lie groupoids and Lie algebroids, volume 213 of London Mathematical Society Lecture Note Series. Cambridge University Press, Cambridge, 2005.

[6] J.E.Marsden, A.Weinstein. Reduction of symplectic manifold with symmetry, Rep. Math. Phys. 5 (1974) no.1 121-130.

[7] R. Montgomery. Canonical formulations of a classical particle in a Yang-Mills field and Wong's equations. Lett. Math.Phys. 8 (1984) 59-67.

[8] J.Śniatycki Geometric Quantization and Quantum Mechanics Springer-Verlag, 1977.

[9] J.Śniatycki On Hamiltonian dynamics of particles with gauge degrees of freedom J.Hadronic, 2, 642-656 (1979).

[10] J.M.Souriau Structure des Systemes Dynamiques Dunod, Paris, 1970.

[11] S.Sternberg Minimal coupling and the symplectic mechanics of a classical particle in the presence of a Yang-Mills field. Proc.Natl.Acad.Sci.USA.,74(12):5253-5254, 1977.

[12] A. Weinstein. A universal phase space for particles in Yang-Mills fields. Lett. Math. Phys., 2(5):417-420, 1977/78.

[13] S.K.Wong. Field and Particle Equations for the Classical Yang-Mills Field and Particles with Isotopic Spin. Nuovo Cimento, 65A: 689-694 (1970). 